\title{
PD-L1 Expression in Colorectal Carcinoma: Immunohistochemical Study
}

\author{
Mehak Gupta*, Mridu Manjari and Harjot Kaur \\ Department of Pathology, SGRD Institute of Medical Sciences and Research, Amritsar
}

\begin{abstract}
Background: Colorectal carcinoma is the most common type of GIT malignancy which is a multifactorial process with etiology encompassing genetic factors, environmental exposures and inflammatory conditions of the digestive tract.

Method: 40 histologically proven cases of colorectal carcinoma were subjected for IHC expression of PD-L1 using Biocare kit. PD-L1 was takenpositive as yellow to brown particles in membrane, cytoplasm or both. It was graded depending upon staining intensity and percentage of the cells andscoring was done by multiplying both and $\geq 3$ was taken as positive score.

Result: Most of the cases were in 6th and 7th decade of life (62.5\%) with M:F ratio being 1.4:1. Most common site involved was rectum. Histologically 29 cases were conventional adenocarcinoma with rest being mucinous and mucin secreting type and most of them being moderately differentiated (70\%). On immunohistochemistry, PD-L1 expression was seen in 20 cases with moderate and strong intensity. But PD-L1 score was positive in 14 cases (35\%). TIL was positive in $57.5 \%$ of the cases. Statistically there was significant correlation between histological type of tumor with PD-L1 ( $p$ value $=0.03$ ) and TIL ( $p$ value $=0.017$ ). Although PD-L1 positivity was insignificant with histological differentiation ( $\mathrm{p}$ value $=0.65$ ) but TIL was significant ( $\mathrm{p}$ value $=0.03$ ).
\end{abstract}

Conclusion: PD-L1 expression is relevant to the prognosis as well as for further CRC therapies whereas the TIL cell positivity has become an independent predictor of patients survival.

Keywords: Colorectal Carcinoma, PD-L1, Tumor Infiltrating Lymphocytes

\section{Introduction}

Colorectal cancer (CRC) is one of the leading causes of cancer-associated mortality worldwide and ranks third, behind prostate cancer and lung cancer for new cases in males and behind breast cancer and lung cancer for new cases in females ( $8 \%$ of all new cancer cases for both). ${ }^{1}$ According to the WHO GLOBOCAN database, there are 1,849,518 estimated new CRC cases and 880,792 CRC-related deaths in 2018. ${ }^{2}$ The global burden of CRC is expected to increase by $60 \%$ to more than 2.2 million new cases and 1.1 million cancer deaths by $2030 .^{3}$ The incidence rates in India are quite low of about 2 to 8 per 100,000 as compared to other countries; ${ }^{4}$ but apart from geographical variations, the incidences are rising rapidly in India. Incidence rate in different subsides varies for age, gender, and race.

It is a multifactorial disease process, with etiology encompassing genetic factors, environmental exposures (including diet), and inflammatory conditions of the digestive tract. Many studies have attributed the increased risk of developing colorectal cancer to living the "Westernized Lifestyle" which encompasses increased dietary fat (particularly rich in animal fat), physical inactivity, obesity (high BMI). ${ }^{1}$ Other causative factors include diabetes, smoking, alcohol intake and genetic susceptibility such as family history, adenomatous polypi, familial polyposis, hereditary non-polyposis colon cancer syndrome and inflammatory bowel diseases, HNF4A, CHDH1, and LAMB1 genes (on chromosomes 20q13, $16 \mathrm{q} 22$, and $7 \mathrm{q} 31$, respectively). Protective role of dietary fibres, aspirin, legumes, fish and supplemental calcium intake on colonic carcinogenesis is also well documented.

Early CRC often has no symptoms, which is why screening is very important in such cases. As a tumor grows, it may present with unexplained anemia, bleeding from the rectum, bloody or dark/black stools, repeated urges to have a bowel movement when the bowel is empty, alternate constipation or diarrhea that lasts for more than a few days, decreased appetite with unintentional weight loss. ${ }^{5}$

Histological types of CRC are mainly adenocarcinoma (90-95\%), others being leiomyosarcoma, lymphoma, melanoma and neuroendocrine tumors, adenosquamous, pure squamous cell carcinoma, spindle cell carcinoma, choriocarcinoma and clear cell carcinoma.

The single most important prognostic indicator of CRC is the extent of the tumor at the time of the diagnosis, the socalled stage. 
Various modalities are used to diagnose CRC including Digital Rectal Examination, Colonoscopy, FNAC etc. But these days Immunohistochemistry and molecular markers are being performed not only for diagnostic purpose but also for predicting the prognosis and target therapy. ${ }^{6}$

Programmed death ligand 1 (PD-L1), also known as CD274 and B7-H1, is a cell surface glycoprotein that plays an important role in the suppression of cellular immune responses. It is a transmembrane protein commonly expressed on the surface of antigen presenting cells and tumor cells. PD-L1 specifically binds to its receptor, PD-1, which is expressed on the variety of immune cells, such as tumor infiltrating lymphocytes, T cells, B cells, monocytes and dendritic cells.?

Studies have demonstrated that binding of tumor PD-L1 to its receptor PD-1 on T cell surface inhibits infiltrating $\mathrm{T}$ cell activation and subsequent lysis of tumor cells. PDL1 expression is detected not only in tumor-infiltrating lymphocytes (TILs), but also present in tumor cells, and its aberrant expression is associated with a poor prognosis in several human tumors. The role of PD-L1 expression in colorectal carcinoma is less clear, with some published studies reporting conflicting results as to whether PD-L1 expression indicates a better or worse prognosis. ${ }^{8}$

\section{Material \& Methods}

The study was conducted on 40 histopathologically proven cases of colorectal cancer received as 16 colectomies and 24 biopsies in a tertiary care center. The tissue was formalin fixed and paraffin embedded and was then stained for haematoxylin and eosin for histopathological typing and grading. All the cases were subjected to immunohistochemistry for PDL1 expression.

Immunohistochemistry was performed using antibodies against PD-L1 (Biocare medical). The antigen retrieval was done using a Decloaker method with $10-\mathrm{mmol}$ citrate buffer at $\mathrm{pH}$ 6.0. Tris buffer was used as wash buffer. Positive control tissue were having colored end product at the site (cytoplasmic and membranous) of target antigen. Negative control tissue section did not have the above colour. PD-L1 presents a positive colour of Yellow to Brown particles in the membrane/ cytoplasm/ both.

Scoring of PD-L1 ${ }^{9}$ : On basis of colour(a) $(0=$ No coloring, 1 $=$ Light yellow, $2=$ Brown Yellow, $3=$ Dark Brown). On the basis of percentage of positive cells (b) $(1=\leq 10 \%$ of the cells, $2=10 \%$ to $50 \%$ of the cells, $3=>50 \%$ of the cells). Integrated score (Immunohistochemical scores, IHS) $=$ Staining intensity(a) $X$ Score of percentage of positive cells(b). [Positive score $=\geq 3$ points].

\section{Results}

The age of the patients varied from 19-82 years of age with maximum number of cases $(62.5 \%)$ in $6^{\text {th }}$ and $7^{\text {th }}$ decade of life with M:F ratio being 1.4:1. Major complaints encountered in the patients were abdominal pain, bleeding per rectum and weight loss that presented mostly in combination of one or the other. Majority of the tumors (27/40 cases) were located in rectum followed by sigmoid colon. Among 40 cases, 16 were colectomies and the rest being biopsies. Majority of the colectomies had tumor size between 2-5 $\mathrm{cm}$ and showed different pattern of tumor growth with maximum being exophytic/polypoidal type $(50 \%)$. The histological profile of CRC comprised maximum number of cases of conventional adenocarcinoma $(72.5 \%)$ with remaining being mucinous adenocarcinoma. 5/40 (12.5\%) cases were well differentiated (Grade I), 28/40 cases (70\%) were moderately differentiated (Grade II) and 7/40 $(17.5 \%)$ cases fell into category of poorly differentiated adenocarcinoma (Grade III). Lymph nodes were recovered in 15 colectomies and of these 8 cases $(53.3 \%)$ showed secondary carcinomatous deposits. [Table 1]

On immunohistochemistry, PD-L1 positive cells were seen in $50 \%(20 / 40)$ of cases, out of which 13 cases $(32.5 \%)$ showed $10-50 \%$ positive cells and 7 cases (17.5\%) showed $<10 \%$ positive cells. Intensity of staining in most of the positive cases varied from moderate $(25 \%)$ to severe $(20 \%)$ with weak $(5 \%)$ in few cases. Although PD-L1 positive cells were seen in 20 cases, but after calculating the score it was positive in 14 cases $(35 \%)$ with score $\geq 3$. $23 / 40$ cases (57.5\%) showed presence of tumor infiltrating lymphocytes (TIL). [Table 1]

On correlating, out of the total 14 PD-L1 score positive cases, 12 cases showed presence of TIL. And out of the 26 PD-L1 score negative cases, 11 cases showed presence of TIL. There was statistically no significant correlation of PD-L1 and TIL with gender [PD-L1 $\mathrm{p}$ value $=0.458$ and TIL $\mathrm{p}$ value $=0.6282$ ], age $[\mathrm{PD}-\mathrm{L} 1 \mathrm{p}$ value $=0.4878$ and TIL $\mathrm{p}$ value $=0.185]$ and tumor size [PD-L1 $\mathrm{p}$ value $=0.69$ and TIL $\mathrm{p}$ value $=0.6143$ ] Although PD-L1 score positivity and TIL was more in rectum and sigmoid colon tumors as compared to tumors of ascending and transverse colon but no significant correlation was seen $\mathrm{p}$ value being 0.14 (PD-L1) and 0.5 (TIL). No definite $\mathrm{p}$ value could be calculated for lymph node status of the tumor. There was statistically significant correlation between histological type of tumor with PD-L1 ( $p$ value $=0.03$ ) and TIL ( $\mathrm{p}$ value $=0.017$ ) as positivity was more in conventional adenocarcinoma and it markedly reduced in mucinous and mucin secreting adenocarcinomas. No statistical significant correlation of PD-L1 score ( $\mathrm{p}$ value $=0.65$ ) and differentiation of tumor was seen but significant correlation of TIL and tumor differentiation was present ( $\mathrm{p}$ value $=0.03$ ). [Table 2] 
Table 1: Patient Demographics and IHC.

\begin{tabular}{|c|c|c|}
\hline PARAMETER & NO OF CASES & PERCENTAGE \\
\hline \multicolumn{3}{|l|}{ Age } \\
\hline$<50$ yrs & 12 & $30 \%$ \\
\hline $51-70 y r s$ & 25 & $62.5 \%$ \\
\hline$>70 y r s$ & 3 & $7.5 \%$ \\
\hline \multicolumn{3}{|l|}{ Gender } \\
\hline Male & 23 & $57.5 \%$ \\
\hline Female & 17 & $42.5 \%$ \\
\hline \multicolumn{3}{|l|}{ Tumour Size } \\
\hline $2-5 \mathrm{~cm}$ & 9 & $56.25 \%$ \\
\hline$>5 \mathrm{~cm}$ & 7 & $43.75 \%$ \\
\hline \multicolumn{3}{|l|}{ Tumour Site } \\
\hline Ascending colon and transverse colon & 7 & $17.5 \%$ \\
\hline $\begin{array}{l}\text { Descending colon, sigmoid colon and its combination with } \\
\text { rectum }\end{array}$ & 13 & $32.5 \%$ \\
\hline Rectum & 20 & $50 \%$ \\
\hline \multicolumn{3}{|l|}{ Histological Type } \\
\hline Conventional adenocarcinoma & 29 & $72.5 \%$ \\
\hline Mucinous and with mucin secreting adenocarcinoma & 11 & $27.5 \%$ \\
\hline \multicolumn{3}{|l|}{ Histological Differentiation } \\
\hline Well & 5 & $12.5 \%$ \\
\hline Moderate & 28 & $70 \%$ \\
\hline Poor & 7 & $17.5 \%$ \\
\hline \multicolumn{3}{|l|}{ Lymph Node } \\
\hline Metastatic & 8 & $53.3 \%$ \\
\hline Reactive & 7 & $46.7 \%$ \\
\hline \multicolumn{3}{|l|}{ PDL-1 Expression } \\
\hline Negative & 20 & $50 \%$ \\
\hline$<10 \%$ & 7 & $17.5 \%$ \\
\hline $10-50 \%$ & 13 & $32.5 \%$ \\
\hline$>50 \%$ & 0 & $0 \%$ \\
\hline \multicolumn{3}{|l|}{ Staining Intensity } \\
\hline Nil & 20 & $50 \%$ \\
\hline Mild & 2 & $5 \%$ \\
\hline Moderate & 10 & $25 \%$ \\
\hline Strong & 8 & $20 \%$ \\
\hline \multicolumn{3}{|l|}{ PDL-1 Score } \\
\hline$<3$ & 26 & $65 \%$ \\
\hline$\geq 3$ & 14 & $35 \%$ \\
\hline \multicolumn{3}{|l|}{ TIL } \\
\hline Positive & 23 & $57.5 \%$ \\
\hline Negative & 17 & $42.5 \%$ \\
\hline
\end{tabular}


Table 2: Correlation Between PDL-1 and TIL.

\begin{tabular}{|c|c|c|c|c|c|c|}
\hline \multirow{2}{*}{ PARAMETER } & \multicolumn{2}{|c|}{ PDL-1 } & \multirow{2}{*}{$\begin{array}{c}p \\
\text { value }\end{array}$} & \multicolumn{2}{|c|}{ TIL } & \multirow{2}{*}{$\begin{array}{c}p \\
\text { value }\end{array}$} \\
\hline & + & - & & + & - & \\
\hline \multicolumn{6}{|l|}{ Age } & \multirow{4}{*}{0.18} \\
\hline$<50$ yrs & 4 & 8 & \multirow{3}{*}{0.48} & 5 & 7 & \\
\hline $51-70 y r s$ & 8 & 17 & & 15 & 10 & \\
\hline$>70 y r s$ & 2 & 1 & & 3 & 0 & \\
\hline \multicolumn{3}{|l|}{ Gender } & \multirow{3}{*}{0.40} & & & \multirow{3}{*}{0.62} \\
\hline Male & 9 & 14 & & 12 & 11 & \\
\hline Female & 5 & 12 & & 11 & 6 & \\
\hline \multicolumn{3}{|l|}{ Tumour Size } & \multirow{3}{*}{0.69} & & & \multirow{3}{*}{0.61} \\
\hline $2-5 \mathrm{~cm}$ & 3 & 6 & & 4 & 5 & \\
\hline$>5 \mathrm{~cm}$ & 3 & 4 & & 4 & 3 & \\
\hline \multicolumn{3}{|l|}{ Tumour Site } & \multirow{4}{*}{0.14} & & & \multirow{4}{*}{0.5} \\
\hline Ascending colon and transverse colon & 4 & 3 & & 5 & 2 & \\
\hline Descending colon, sigmoid colon and its combination with rectum & 2 & 11 & & 6 & 7 & \\
\hline Rectum & 8 & 12 & & 12 & 8 & \\
\hline \multicolumn{3}{|l|}{ Histological Type } & \multirow{3}{*}{0.03} & & & \multirow{3}{*}{0.01} \\
\hline Conventional adenocarcinoma & 13 & 16 & & 20 & 9 & \\
\hline Mucinous and with mucin secreting adenocarcinoma & 1 & 10 & & 3 & 8 & \\
\hline \multicolumn{3}{|l|}{ Histological Differentiation } & \multirow{4}{*}{0.65} & & & \multirow{4}{*}{0.03} \\
\hline Well & 1 & 4 & & 4 & 1 & \\
\hline Moderate & 11 & 17 & & 18 & 10 & \\
\hline Poor & 2 & 5 & & 1 & 6 & \\
\hline
\end{tabular}

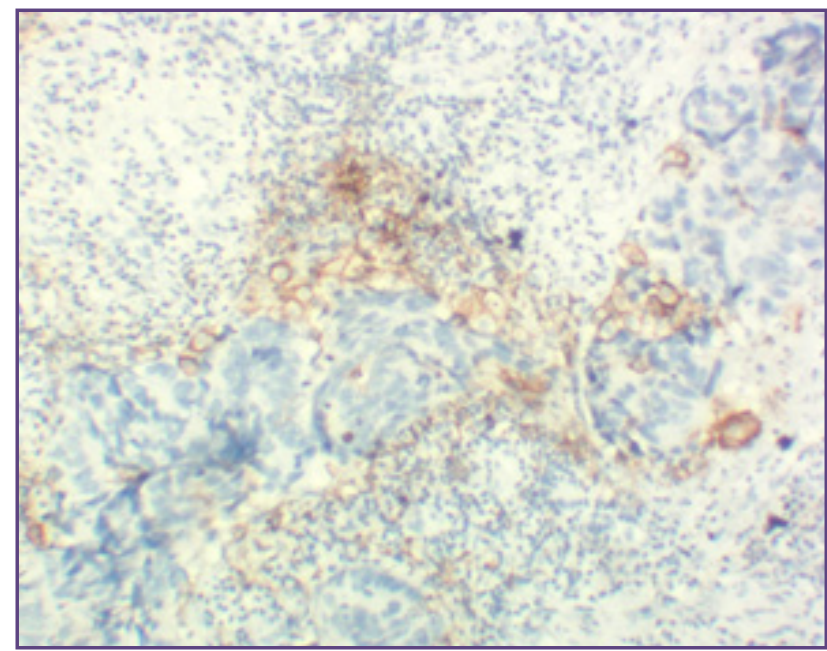

Fig. a) PD-L1 Positivity (Membranous, 400X).

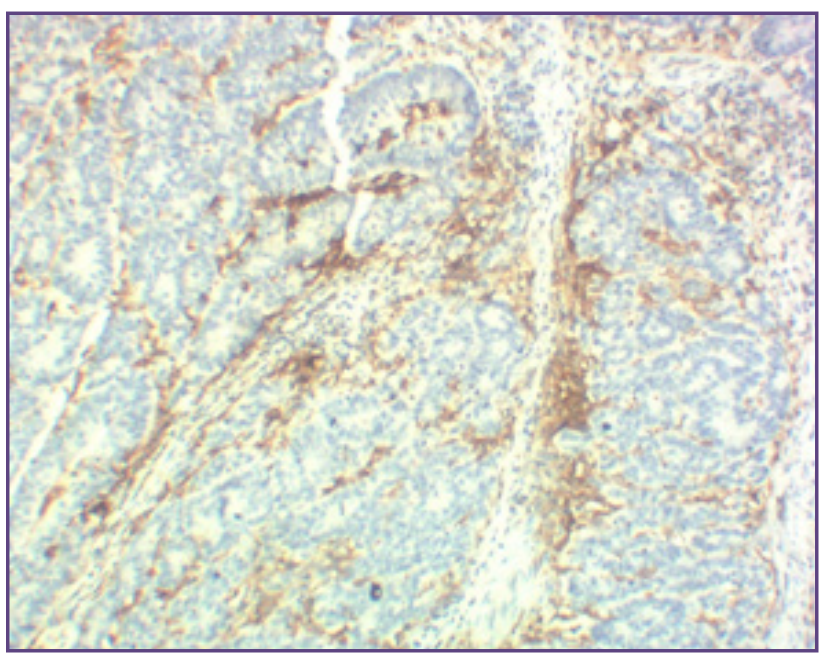

Fig. b)PD-L1 Positivity (Bothmembranous/ Cytoplasmi C, 400X).

Annals of Pathology and Laboratory Medicine, Vol. 7, Issue 6, June, 2020 


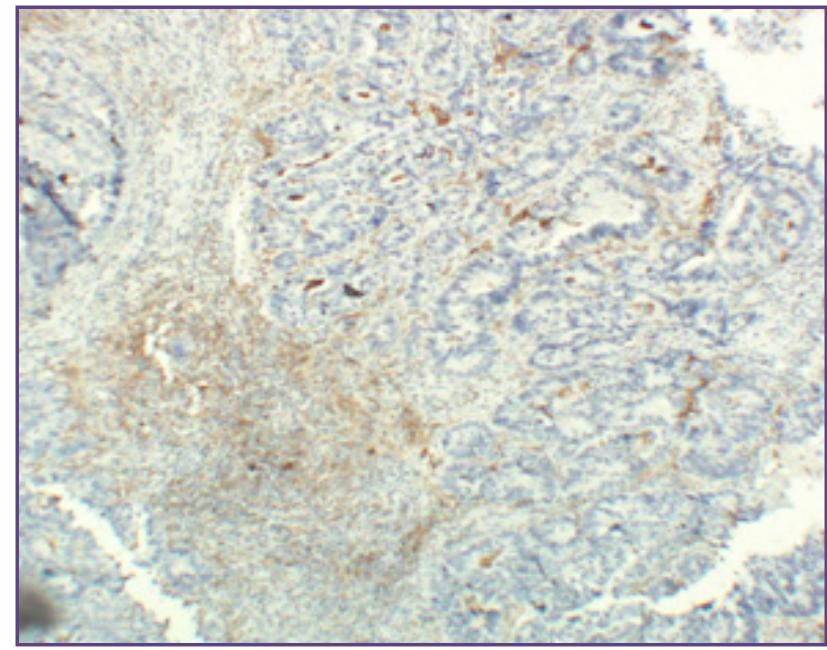

Fig. c) Tumour Infiltrating Lymphocytes (TIL) Positivity.

\section{Discussion}

Colorectal cancer (CRC) is one of the most frequent malignancies in the world. Despite major advances in diagnosis and treatment of the disease, overall mortality is still high and needs further efforts to reduce cancer related death. ${ }^{10}$ Although the tumor is diagnosed histopathologically on light microscopy but various immunological markers are expressed by CRC and depending upon them the treatment and prognosis differs. PD-L1 is an immunoinhibitory molecule that suppresses the activation of $\mathrm{T}$ cells, leading to progression of tumors. The prognostic value in CRC remains unclear. PD-L1 positive TIL is an independent prognostic factor in CRC patients especially for MMR proficient subgroup. ${ }^{11}$

Present study showed maximum number of cases in the age group of 51-70 years (62.5\%) with $\mathrm{M}: \mathrm{F}$ ratio being 1.4:1 in concordance with White et al. ${ }^{12}$ PD-L1 score positivity was also more in males in this age group but no significant correlation seen. Same findings has been reported by Shen et al. ${ }^{13}$

Although immunoexpression of PD-L1 was seen in $50 \%(20 / 40)$ of the cases with maximum cases showing $10-50 \%$ expression of positive cells but PD-L1 score was positive in $35 \%(14 / 40)$ of the case. TIL was positive in $23 / 40$ cases $(57.5 \%)$. Mathew et al concluded 16/181 cases ( $9 \%$ ) to be positive for PD-L1 expression using the 5\% cutoff (PD-L1 score 1-2) and 10/16 (62\%) PD-L1-positive tumors contained a high number of CD8-positive tumorinfiltrating lymphocytes. ${ }^{14}$ Ho kim et al showed PDL-1 positivity in tumour cells and immune cells to be $12.5 \%$ and $29.8 \%$ MSI-H CRCs, respectively and occasionally overlapped $(\mathrm{n}=12 ; 5.8 \%) .{ }^{15}$ Shingo et al showed variable PDL-1 expression in 54/454 CRC (12\%). ${ }^{16}$
Rectum was involved in 27 cases with rest being in different parts of colon and most of them were diagnosed as conventional adenocarcinoma moderately differentiated (70\%) followed by poorly differentiated. Similar results have been seen with various researchers. ${ }^{17,18,19}$ PD-L1 positivity was also more in rectal and sigmoid tumors with insignificant correlation. But Anna et al showed significant association of PD-L1 and TIL with right sided tumors. ${ }^{20}$

PD-L1 positivity in tumor cell and TIL positivity decreased with mucinous feature (PD-L1: 1 case; TIL: 3 case) in $\mathrm{CRC}$ as compared to conventional adenocarcinoma (PDL1:13 case; TIL:20 case) leading to significant correlation which is in concordance with study by Ho Kim et al which showed PD-L1 positvity in tumour cells $(65 \%)[\mathrm{p}=0.013]$ and/or immune cells $(55 \%)[\mathrm{p}=0.022]$ subset of MSI-H CRCs was significantly associated with non mucinous type tumors. ${ }^{15}$ Colorectal mucinous adenocarcinoma has a dismal prognosis compared to conventional one.

As the grade increased, PD-L1 score as well as TIL positivity decreased. Hence there was significant correlation of TIL with histological differentiation. But insignificant correlation of PD-L1 score and histological differentiation was found. But Shingo et al showed significant association between PD-L1 expression and poor differentiation. ${ }^{16}$

Thus study showed higher expression of TIL to be significantly correlated with good prognosis (as an independent prognostic factor). This is in concordance with Yaqi et al which showed higher expressions of PD-1 and PD-L1 correlating with better prognosis of CRC patients and TILs-PD-1 to be an independent prognostic factor for OS and DFS of CRC patients, especially for MMRproficient subgroup. ${ }^{11}$

Now-a-days CRC, on the basis of prognostic and therapeutic purposes, is divided into 2 types, on the basis of DNA mismatch repair (MMR) status - MMR proficient and MMR deficient (found in other types such as HNPCC, polyps etc). ${ }^{21}$

Several clinical trials have been conducted on monoclonal antibodies (MAbs) against PD-1 in CRC including combination therapies of cytotoxic agents and targeted therapies, particularly targeting epidermal growth factor receptor (EGFR) by Cetuximab or Panitumumab and vascular endothelial growth factor receptor (VEGFR) pathway by Bevacizumab. It recognized the importance of anti-PD-1 therapy for MSI (Microsatellite instability) tumors among CRC subtypes. ${ }^{22}$

CRC was further investigated by a small phase 2 trial of Pembrolizumab and Nivolumab (human $\mathrm{mAb}$ targeting PD-1). It gave the first indication that PD-1 targeting of 
colorectal carcinoma in metastatic disease significantly benefited patients with MSI tumors when compared with those with MSS (microsatellite stable) disease. It also indicated that PD-L1 expression (assessed by IHC) was strongly associated with MSI (dMMR), suggesting that expression of PD-L1 may be a useful predictive biomarker of response to PD-1 immune checkpoint targeting. This study showed that MMR status predicted clinical benefit of immune checkpoint blockade with both agent with enhanced responsiveness in MSI CRC. ${ }^{23}$

\section{Conclusion}

PD-L1 expression is associated with signatures of serrated pathway of colorectal carcinogenesis including BRAF mutation, microsatellite instability and is more common with MMR proficient as compared to MMR deficient CRCs, correlates with overall survival rate of CRC. PD-L expression is suggestive to be involved in downregulating anti-tumor immunity and thus promoting further tumor growth and metastasis in patients with CRC. Therapeutically, significant correlation has been observed between PD-L1 overexpression, infiltration of PD-1 CD8+ lymphocytes and is strongly demonstrated in mismatch repair (MMR) proficient tumors. It has been observed that CRC patients which show immune related objective response to anti PD-1 treatment are usually MMR deficient, thus drugs Pembrolizumab and Nivolumab may play a role in these cases if CRC is MMR deficient, although positivity of TIL plays as an independent prognostic factor.

\section{Funding}

Nil

\section{Conflicts of Interests}

There are no conflicts of interest.

\section{References}

1. Marley AR, Nan H. Epidemiology of Colorectal Cancer. Int J Mol Epidemiol Genet. 2016;7(3):105-14

2. Wong M, Ding H, Wang J, Chan P, Huang J. Prevalence and Risk Factors of Colorectal Cancer in Asia. Intest Res. 2019;17(3):317-29

3. Arnold M, Sierra MS, Laversanne M, Soerjomataram I, Jemal A, Bray F. Global patterns and trends in Colorectal Cancer Incidence and Mortality. Gut. 2017 Apr;66(4):683-91

4. Parkin D, Pisani P, Ferlay J. Global Cancer Statistics. Int J Cancer. 1999 Jan;80(1):33- 64.

5. American Cancer Society. Colorectal Cancer Facts \& Figures 2017-2019 [Internet]. Atlanta: American Cancer Society; 2017. Available form: https://www.cancer.org/ content/dam/cancer-org/research/cancer-facts-and-statistics/ colorectal-cancer-facts-and-figures/colorectal-cancer-factsand-figures-2017-2019.pdf
6. Locker GY, Hamilton S, Harris J. ASCO 2006 update of recommendations for the use of tumor markers in gastrointestinal cancer. J Clin Oncol. 2006 Nov;24(11):5313-27.

7. Wu Y, Chen W, Xu ZP, Gu W. PD-L1 Distribution and perspective for cancer immunotherapy- Blockade, Knockdown, or Inhibition. Front Immunol. 2019 Aug; 10:2022

8. Wang X, Teng F, Kong L, Yu J. PD-L1 Expression in Human Cancers and Its Association with Clinical Outcomes. OncoTargets and Therapy. 2016;9:5023-39.

9. Lou J, Zhou Y, Huang J, Qian X. Relationship between PD-L1 expression and clinical characteristics in patients with breast invasive ductal carcinoma. Open Med (Wars) [Internet]. 2017 Sep [cited 2017 Nov];12:288-92. Available from: https:/www.ncbi.nlm.nih.gov/pmc/articles/PMC5588755/ DOI: $10.1515 / \mathrm{med}-2017-0042$.

10. Gasser M, Koenigshausen M, Grimm MR, Stein C, Grimmig $\mathrm{T}$, et al. Clinical Significance and Therapeutic Potential of the Programmed Death Ligand-1 (PD-L1) and PD-L2 Expression in Human Colorectal Cancer. Cancer Sci Ther. 2017 Aug;9:566-73.

11. Li Y, Liang L, Dai W, Cai G, Xu Y, Li X, et al. Prognostic impact of PD-1 and PD-L1 expression in cancer cells and tumour infiltrating lymphocytes in colorectal cancer. Mol Cancer. 2016 Aug;15(1):55

12. White A, Ironmonger L, Steele RJ, Ormiston-Smith N, Crawford C, Seims A. A review of sex-related differences in colorectal cancer incidence, screening uptake, routes to diagnosis, cancer stage and survival in the UK. BMC cancer. 2018;18:906.

13. Shen Z, Gu L, Mao D, Chen M, Jin R. Clinicopathological and Prognostic Significance of PD-L1 Expression in Colorectal Cancer: a Systematic Review and Meta analysis. World J Surg Oncol. 2019 Jan;17:4.

14. Rosenbaum MW, Bledsoe JR, Oyarvide VM, Huynh T, Gand Kenudson MM. PD-L1 expression in colorectal cancer is associated with microsatellite instability, BRAF mutation, medullary morphology and cytotoxic tumor-infiltrating lymphocytes. Mod Pathol. 2016 Sep;29(9):1104-12.

15. Kim JH, Park HE, Cho N, Lee SH, Kang GH. Characterisation of PDL-1 positive subsets of microsatellite unstable colorectal cancers. Br J Cancer. 2016 Jul;115:490-96

16. Inaguma $\mathrm{S}$, Lasota J, Wang Z, Felisiak-Golabek A, Ikeda H, Miettinen M. Clinicopathologic profile, immunophenotype, and genotype of CD274(PDL-1)- positive colorectal carcinomas. Mod Pathol. 2017 Feb;30(2):278-85.

17. Sharma D, Singh G. Clinico-pathological profile of colorectal cancer in first two decades of life: A retrospective analysis from tertiary health center. Indian $\mathrm{J}$ Cancer 2017;54:397-400.

18. Fleming M, Ravula S, Tatishchev FL, Wang HL. Colorectal carcinoma: Pathological aspects. J Gastrointest Oncol. 2012 Sep;3(3):153-73. 
19. Manxhuka-Kerliu S, Telaku S, Ahmetaj H, Baruti A, Loxha S, Kerliu A. Colorectal cancer: prognostic values. Bosn J Basic Med Sci. 2009;9(1):19-24

20. Valentini AM, Pinto FD, Cariola F, Guerra V, Gianluigi G, Caruso ML et al. PDL-1 expression in colorectal cancer defines three subsets of tumour immune microenvironments. Oncotarget. 2018 Feb; 9(9): 8584-96.

21. Boland CR, Goel A. Microsatellite instability in colorectal cancer. Gastroenterology. 2010 Jun; 138(6):2073-87.
22. Yaghoubi N, Soltani A, Ghazvini K, Hassanian SM, Hashemy SI. PD-1/PDL-1 blockade as a novel treatment for colorectal cancer. Biomedicine and Pharmacotherapy. $2019 ; 110: 312-8$

23. Jacobs J, Smits E, Lardon F, Pauwels P, and Deschoolmeester V. Immune Checkpoint Modulation in Colorectal Cancer: What's New and What to Expect .J Immunol Res. 2015 Oct;2015:158038.

*Corresponding author:

Dr. Mehak Gupta, 23-S, Swaran Vihar Behind Aggarsain Charitable Hospital, Patiala, Punjab - 14007, India

Phone: +918360936282

Email: mehak2891@gmail.com

Date of Submission : 30/11/2019

Date of Acceptance : 03/04/2020

Financial or other Competing Interests: None.

Date of Publication : 27/06/2020 